\title{
A Thermoelastic Piezoelectric Fixed Rod Exposed to an Axial Moving Heat Source via a Dual-Phase-Lag Model
}

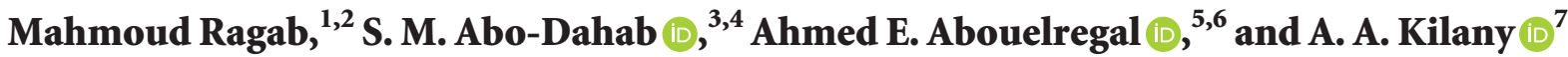 \\ ${ }^{1}$ Department of Information Technology, Faculty of Computing and Information Technology, King Abdulaziz University, \\ Jeddah, Saudi Arabia \\ ${ }^{2}$ Department of Mathematics, Faculty of Science, Al-Azhar University, Cairo, Egypt \\ ${ }^{3}$ Department of Mathematics, Faculty of Science, South Valley University, Qena 83523, Egypt \\ ${ }^{4}$ Department of Computer Science, Faculty of Computers and Information, Luxor University, Luxor, Egypt \\ ${ }^{5}$ Department of Mathematics, Faculty of Science, Mansoura University, Mansoura 35516, Egypt \\ ${ }^{6}$ Department of Mathematics, College of Science and Arts, Jouf University, Al-Qurayyat, Saudi Arabia \\ ${ }^{7}$ Department of Mathematics, Faculty of Science, Sohag University, Sohag, Egypt
}

Correspondence should be addressed to A. A. Kilany; arabyatef@yahoo.com

Received 22 January 2021; Revised 15 February 2021; Accepted 20 April 2021; Published 22 July 2021

Academic Editor: Dan Selisteanu

Copyright (c) 2021 Mahmoud Ragab et al. This is an open access article distributed under the Creative Commons Attribution License, which permits unrestricted use, distribution, and reproduction in any medium, provided the original work is properly cited.

\begin{abstract}
Piezoelectric ceramics are used more in the active control of noise and vibration with sensors and actuators. As a result, a onedimensional homogeneous thermoelastic fixed piezoelectric rod subjected to a moving heat flow has been considered. The heating rod is affected by three fields, namely, thermal, mechanical, and electric potential. In order to design a reliable smart structure, this study is necessary and the thermopiezoelectric behavior of piezoelectric ceramics must be understood clearly. The Laplace transform method is applied to acquire the distributions of stress, deformation, and temperature fields. The effect of heat source velocity and phase lag parameters on the considered physical fields is displayed graphically, and the results are compared with other models of thermoelasticity. The present findings could find possible applications for designing receiving portions of transformers of Rosen type for voltage magnification.
\end{abstract}

\section{Introduction}

Normally, in recent decades, more consideration has been given to the thermoelasticity field due to its applied aspects in various fields, especially geology, geophysics, structures, biology, physics, acoustics, plasma, and so forth. The generalized thermoelasticity theory is one of the improved forms of the classical thermoelasticity and has been modified to remove the defects of the classical coupled thermoelasticity. The modified thermoelasticity models were introduced by Lord and Shulman [1], Green and Lindsay [2], and Green and Naghdi [3] as well as by Tzou [4], Tzou [5], and Tzou [6]. On the other hand, many works, in literature, have applied generalized thermoelasticity theories to investigate the thermoelastic response of solid materials [7-12].
Piezoelectric materials are an important kind of smart material, as they are able to cause strain or generate electrical energy. Piezoelectric materials are essential materials that have the ability to convert electrical energy into mechanical energy to stimulate strain and act as an engine when these materials are powered by an electric field. Mostly, piezoelectric devices operate in a thermoelastic coupling medium. Often, piezoelectric devices and piezoelectric materials operate in a medium with pyroelectric and thermoelastic coupling.

To illustrate the thermopiezoelectric interaction, various works have been carried out [13-25]. Abo-Dahab et al. [26] discussed the electromagnetothermoelastic medium with diffusion and voids in the context of Lord-Shulman or dualphase-lag models under influence of rotation and gravity. 
A reverse problem of including interfaces in a piezoelectric structure was proposed by Nanthakumar et al. [27] in order to overcome this algorithm. The material interfaces are implied by the level sets defined by regularization with total variation penalty words. The reverse issue is iteratively solved, and, for the study of each iteration, the extended finite element approach is used. For three-dimensional structures, the wording is presented, and inclusions of different materials are recognized by multiple level sets.

In order to mathematically model various phenomena in science and engineering, partial differential equations (PDEs) are important. To solve these problems is a key step in achieving accurate understanding of the conduct of natural and engineered structures. In general, analytical methods are typically not sufficient to solve PDEs representing real systems to an appropriate degree. One must use methods of choice. The most popular alternative is likely the finite element approach for engineering problems (FEM). But strong alternatives are also available, such as mesh-free methods and the Isogeometric Analysis (IGA). The basic idea is to approximate the PDE solution with specially constructed functions with desired properties. Samaniego et al. [28] have explored an alternative for approximation Deep Neural Networks (DNNs). In that work, the emphasis is on mechanical problems, and the PDE energy format is analyzed. For a computer learning to solve a mechanical problem, the energy of a mechanical system seems to be a normal loss function.

Hamdia et al. [29] have used sensitivity analysis to define crucial parameters affecting flexoelectric materials' energy conversion factor (ECF). The governing flexoelectricity equations are modeled on a NURBS-based IGA formula that uses their higher-order continuity and thus avoids a complex combined formulation. The input parameters examined include model and material characteristics, and sampling has taken place in the likelihood region using the Latin hypercube sampling (LHS) method. Khalil et al. [30] investigated electromagnetic field and initial stress on a photothermal semiconducting voids medium under thermoelasticity theories. Abo-Dahab et al. [33-37] studied the effect of relaxation times in different mediums with several variables. Alotaibi et al. [38] used fractional calculus of thermoelastic P-Waves reflection under influence of gravity and electromagnetic fields. Abd-Alla et al. [39] illustrated the effect of several fields on a generalized thermoelastic medium with voids in the context of Lord-Shulman or dualphase-lag models.

The Laplace transform method is simple to describe. Given an initial value problem (IVP), apply the Laplace transform operator to both sides of the differential equation. This will transform the differential equation into an algebraic equation whose unknown, $F(s)$, is the Laplace transform of the desired solution. Once you solve this algebraic equation for $F(s)$, take the inverse Laplace transform of both sides; the result is the solution to the original IVP.

In addition, the method used in this research is conceptually simple and easy to program. This method is effective in meeting the requirements of automatic digital computation in an efficient manner for two reasons. First, it is fast (economical) on digital computers now available in contrast to other more complicated methods of numerical quadrature. Second, the method is conceptually simple and requires a minimum of programming effort because the resultant inverse function is given as a Fourier cosine series with the coefficients being appropriate values of the forward transform. Therefore, one avoids the use of involved algorithms which are encountered in methods using orthogonal functions.

In the current investigation, the response of a thermoelastic piezoelectric fixed rod is investigated. Also, the rod exposed to a heat source moves over its axis. The problem is expressed in the context of Tzou [5] model. By using the Laplace transform and the Laplace numerical inversion, we solved the problem. The influence of speed of the moving heat source and the phase lags on displacement, stress, and temperature is explained.

\section{Basic Equations}

The governing equations of thermopiezoelectric materials without body forces and free charge are as follows [16, 21]:

Strain-displacement relations:

$$
\varepsilon_{i j}=\frac{1}{2}\left(u_{i, j}+u_{j, i}\right), \quad i, j=1,2,3
$$

Stress-strain-temperature and electric field relations:

$$
\begin{aligned}
& \sigma_{i j}=c_{i j k l} \varepsilon_{k l}-\gamma_{i j}\left(T-T_{0}\right)-e_{j i k} D_{k}, \\
& E_{i}=e_{i j k} \varepsilon_{k j}+\epsilon_{i k} D_{k}+p_{i}\left(T-T_{0}\right), \quad i, j, k=1,2,3 .
\end{aligned}
$$

The motion equation:

$$
\sigma_{j i, j}=\rho \ddot{u}_{i}, \quad i, j=1,2,3 .
$$

The heat equation proposed by Tzou [6]:

$$
\left(1+\tau_{\theta} \frac{\partial}{\partial t}\right)\left(K_{i j} T_{j}\right)_{i}=\left(\delta+\tau_{q} \frac{\partial}{\partial t}\right)\left(\rho C_{E} \frac{\partial T}{\partial t}+T_{0} \gamma_{i j} \frac{\partial u_{i, j}}{\partial t}-T_{0} p_{i} \frac{\partial \phi_{i}}{\partial t}-Q\right) .
$$

Electric and Gauss equations: 


$$
\begin{aligned}
D_{i, i} & =0, \\
E_{i} & =-\dot{\phi}_{i},
\end{aligned}
$$

where $\sigma_{i j}$ represents the stress tensor components, $\varepsilon_{i j}$ represents the strain tensor components, $E_{i}$ represents the electric field vector components, $K_{i j}$ represents the thermal conductivity tensor, $c_{i j k l}$ represents the elastic constants, $\gamma_{i j}$ represents the thermal moduli, $\phi$ represents the function of electric potential, $e_{j i k}$ represents the piezoelectric moduli, $T$ represents the temperature increment, $T_{0}$ represents the initial temperature, $D_{i}$ represents the electric displacement components, $u_{i}$ represents the displacement vector components, $\rho$ represents the mass density, and $\varepsilon_{i k}$ represents the electric permittivity. Also, $C_{E}$ refers to the specific heat, $Q$ refers to the applied heat source, $p_{i}$ refers to the pyroelectric constants, $\tau_{q}$ refers to the phase lag of heat flux, and $\tau_{\theta}$ refers to the dual-phaselag parameter. Equations (1)-(5) are the field equations of the generalized linear thermoelastic-piezoelectric applicable for the following:

(i) Dynamical coupled thermoelastic-piezoelectric (CD theory), when $\tau_{\theta}=0=\tau_{q}$

(ii) Generalized piezothermoelasticity (LS), when $\tau_{\theta}=0, \tau_{q}=\tau_{0}>0$, where $\tau_{0}$ is the relaxation time

(iii) Generalized linear piezothermoelasticity without energy dissipation (GN model), when $\tau_{q}=1$ and $\tau_{\theta}=\delta=0$

(iv) Generalized piezothermoelasticity with two different phase lags (DPL model), when $\tau_{q} \geq \tau_{\theta}>0$

\section{Problem Formulation}

We consider the direction of polarization of a piezoelectric rod finite length $L$ that is parallel to the axial direction of the rod. Also, the two ends of rod are assumed to be fixed and thermally insulated. Initially, the rod is not deformed and at rest. The $x$-axis coincides with the axial of the rod. The temperature $T$ and the displacement component $u$, for a one-dimensional problem, can be written as

$$
\begin{aligned}
T & =T(x, t), \\
u_{x} & =u(x, t) .
\end{aligned}
$$

The strain component $e$ is given by

$$
e=e_{x x}=\frac{\partial u}{\partial x}
$$

We consider that there are no free charges within the piezoelectric rod. Then, Gauss's law gives

$$
\operatorname{div}(D)=0,
$$

which can be rewritten as

$$
\frac{\partial D}{\partial x}=0
$$

This leads to $D=D(t)$. For simplification, we will consider $D$ as constant along the piezoelectric rod. Equations (2), (3), and (5) may be reduced to

$$
\sigma_{x x}=\sigma=(\lambda+2 \mu) \frac{\partial u}{\partial x}-\gamma\left(T-T_{0}\right)-e_{1} D
$$

$(\lambda+2 \mu) \frac{\partial^{2} u}{\partial x^{2}}-\gamma \frac{\partial T}{\partial x}=\rho \frac{\partial^{2} u}{\partial t^{2}}$,

$$
K\left(1+\tau_{\theta} \frac{\partial}{\partial t}\right) \frac{\partial^{2} T}{\partial x^{2}}=\left(1+\tau_{q} \frac{\partial}{\partial t}\right)\left(\rho C_{E} \frac{\partial T}{\partial t}+T_{0} \gamma \frac{\partial u}{\partial x \partial t}-Q\right)
$$

where $\gamma=(3 \lambda+2 \mu) \alpha_{t}, \alpha_{t}$ is the thermal expansion coefficient, $K$ is the thermal conductivity, and $e_{1}$ is the piezoelectric constant. To obtain the dimensionless form of the basic equations, we introduce the following variables:

$$
\begin{aligned}
\left\{x^{*}, u^{*}\right\} & =c_{0} \eta\{x, u\}, \\
t^{*} & =c_{0}^{2} \eta t, \\
\left\{\tau_{q}^{*}, \tau_{\theta}^{*}\right\} & =c_{0}^{2} \eta\left\{\tau_{q}, \tau_{\theta}\right\}, \\
T^{*} & =\frac{T-T_{0}}{T_{0}}, \\
\sigma^{*} & =\frac{\sigma}{\lambda+2 \mu}, \\
D^{*} & =\frac{e_{1} D}{\lambda+2 \mu}, \\
\eta & =\frac{\rho C_{E}}{K} \\
c_{0}^{2} & =\frac{\lambda+2 \mu}{\rho} . \\
Q^{*} & =\frac{\gamma Q}{K c_{0}^{2} \eta^{2}(\lambda+2 \mu)},
\end{aligned}
$$

Introducing equation (14) into (11)-(13), we get (dropping the asterisks)

$$
\begin{aligned}
\sigma & =\frac{\partial u}{\partial x}-b T-D, \\
\frac{\partial^{2} u}{\partial x^{2}}-b \frac{\partial T}{\partial x} & =\frac{\partial^{2} u}{\partial t^{2}} \\
\left(1+\tau_{\theta} \frac{\partial}{\partial t}\right) \frac{\partial^{2} T}{\partial x^{2}} & =\left(1+\tau_{q} \frac{\partial}{\partial t}\right)\left(\frac{\partial T}{\partial t}+g \frac{\partial u}{\partial x \partial t}-Q\right),
\end{aligned}
$$

where 


$$
\begin{aligned}
& b=\frac{\gamma T_{0}}{\lambda+2 \mu}, \\
& g=\frac{\gamma}{\rho C_{E}} .
\end{aligned}
$$

The nondimensional form of the moving heat source $Q$ can be taken as

$$
Q=Q_{0} \delta(x-v t)
$$

where $\delta$ is the delta function, $Q_{0}$ is the heat source strength, and $v$ is the velocity of $Q$ along the positive direction of the $x$-axis.

\section{Initial and Boundary Conditions}

We consider the initial conditions to be

$$
\begin{array}{cl}
\left.u(x, t)\right|_{t=0}=\left.\frac{\partial u(x, t)}{\partial t}\right|_{t=0}=0, & 0 \leq x \leq l, \\
\left.T(x, t)\right|_{t=0}=\left.\frac{\partial T(x, t)}{\partial t}\right|_{t=0}=0, & t=0,0 \leq x \leq l .
\end{array}
$$

As the rod is fixed and thermally insulated, the boundary conditions are as follows:

$$
\begin{gathered}
u(0, t)=u(l, t)=0, \\
\frac{\partial T(0, t)}{\partial x}=\frac{\partial T(l, t)}{\partial x}=0 .
\end{gathered}
$$

\section{Laplace Transform}

The Laplace transform of any function $f(t)$ is given by

$$
\mathfrak{E}\{f(t)\}=\bar{f}(s)=\int_{0}^{\infty} e^{-s t} f(t) \mathrm{d} t, \quad \operatorname{Re}(s)>0 .
$$

After using the Laplace transform, we obtain

$$
\begin{aligned}
\bar{\sigma} & =\frac{\mathrm{d} u}{\mathrm{~d} x}-b \bar{T}-\frac{\bar{D}}{s}, \\
\left(\frac{\mathrm{d}^{2}}{\mathrm{~d} x^{2}}-s^{2}\right) \bar{u} & =b \frac{\mathrm{d} \bar{T}}{\mathrm{~d} x}, \\
\left(\frac{\mathrm{d}^{2}}{\mathrm{~d} x^{2}}-q s\right) \bar{T} & =q s g \frac{\mathrm{d} u}{\mathrm{~d} x}-\alpha q \exp \left(-\frac{s}{v} x\right),
\end{aligned}
$$

where

$$
\begin{aligned}
& \alpha=\frac{Q_{0}}{v}, \\
& q=\frac{\left(1+\tau_{q} s\right)}{\left(1+\tau_{\theta} s\right)} .
\end{aligned}
$$

The boundary conditions (19) and (20) are also transformed to

$$
\begin{gathered}
\bar{u}(0, s)=\bar{u}(l, s)=0, \\
\frac{\mathrm{d} \bar{T}(0, s)}{\mathrm{d} x}=\frac{\mathrm{d} \bar{T}(l, s)}{\mathrm{d} x}=0 .
\end{gathered}
$$

\section{Solution in the Transformed Field}

Eliminating $\bar{T}$ between (23) and (24), we have

$$
\left(\frac{\mathrm{d}^{4}}{\mathrm{~d} x^{4}}-\left(s^{2}+s q(1+g b)\right) \frac{\mathrm{d}^{2}}{\mathrm{~d} x^{2}}+q s^{3}\right) \bar{u} \frac{b \alpha s q}{v} \exp \left(-\frac{s}{v} x\right) .
$$

The characteristic equation of (28) takes the form

$$
m^{4}-\left(s^{2}+s q(1+g b)\right) m^{2}+q s^{3}=0 .
$$

The roots $m_{1}^{2}$ and $m_{2}^{2}$ of equation (29) satisfy the relations

$$
\begin{aligned}
m_{1}^{2}+m_{2}^{2} & =\left(s^{2}+s q(1+g b)\right), \\
m_{1}^{2} m_{2}^{2} & =q s^{3},
\end{aligned}
$$

where $m_{1}$ and $m_{2}$ are given by

$$
m_{1,2}^{2}=\frac{1}{2}\left(\left(s^{2}+s q(1+g b)\right) \pm \sqrt{\left(s^{2}+s q(1+g b)\right)^{2}-4 q s^{3}}\right) \text {. }
$$

Solving equation (28), we get

$$
\bar{u}=A_{1} e^{-m_{1} x}+A_{2} e^{m_{1} x}+A_{3} e^{-m_{2} x}+A_{4} e^{m_{2} x}+A_{5} \exp \left(-\frac{s}{v} x\right),
$$

where $A_{i},(i=1,2,3,4)$ all are parameters and

$$
A_{5}=\frac{b \alpha s q v^{3}}{s^{4}-\left(s^{2}+s q(1+g b)\right) s^{2}+v^{4} q s^{3}} .
$$

Likewise, the function $\bar{T}$ satisfies the following equation:

$$
\left(\frac{\mathrm{d}^{4}}{\mathrm{~d} x^{4}}-\left(s^{2}+s q(1+g b)\right) \frac{\mathrm{d}^{2}}{\mathrm{~d} x^{2}}+q s^{3}\right) \bar{T}=\frac{\alpha s^{2} q\left(1-v^{2}\right)}{v^{2}} \exp \left(-\frac{s}{v} x\right) .
$$

The solution to $\bar{T}$ is given from (34) as

$$
\bar{T}=B_{1} e^{-m_{1} x}+B_{2} e^{m_{1} x}+{ }_{1} B_{3} e^{-m_{2} x}+{ }_{1} B_{4} e^{m_{2} x}+B_{5} \exp \left(-\frac{s}{v} x\right),
$$

where $B_{i},(i=1,2,3,4)$ are some parameters that can be determined from the boundary conditions.

Substituting $\bar{u}$ from equation (32) and $\bar{T}$ from equation (35) into (23), we can find the following relationship:

$$
\begin{aligned}
B_{i} & =(-1) \frac{{ }_{i} m_{i}^{2}-s^{2}}{m_{i} b} A_{i}, \quad i=1,2,3,4, \\
B_{5} & =-\frac{s\left(1-v^{2}\right)}{b v} A_{5} .
\end{aligned}
$$


In order to determine the parameters $A_{i},(i=1,2,3,4)$, we need to apply boundary conditions (26) and (27).

$$
\begin{gathered}
A_{1}+A_{2}+A_{3}+A_{4}=-A_{5}, \\
A_{1} e^{-m_{1} l}+A_{2} e^{m_{1} l}+A_{3} e^{-m_{2} l}+A_{4} e^{m_{2} l}=-A_{5} \exp \left(-\frac{s}{v} l\right), \\
-m_{1} B_{1}+m_{1} B_{2}-m_{2} B_{3}+m_{2} B_{4}=\frac{s}{v} B_{5}, \\
-m_{1} B_{1} e^{-m_{1} l}+m_{1} B_{2} e^{m_{1} l}-m_{2} B_{3} e^{-m_{2} l}+m_{2} B_{4} e^{m_{2} l}=\frac{s}{v} B_{5} \exp \left(-\frac{s}{v} l\right) .
\end{gathered}
$$

Solving equations (40)-(43), $A_{i}(i=1,2,3,4)$ can be expressed as

$$
\begin{aligned}
& A_{1}=\frac{\left(m_{2}^{2}-(s / v)^{2}\right)\left(\exp \left(m_{1} l\right)-\exp (-(s / v) x l)\right)}{2\left(m_{1}^{2}-m_{2}^{2}\right) \sinh \left(m_{i} l\right)} A_{5}, \\
& A_{2}=-\frac{\left(m_{2}^{2}-(s / v)^{2}\right)\left(\exp \left(-m_{1} l\right)-\exp (-(s / v) x l)\right)}{2\left(m_{1}^{2}-m_{2}^{2}\right) \sinh \left(m_{1} l\right)} A_{5}, \\
& A_{3}=-\frac{\left(m_{1}^{2}-(s / v)^{2}\right)\left(\exp \left(m_{2} l\right)-\exp (-(s / v) x l)\right)}{2\left(m_{1}^{2}-m_{2}^{2}\right) \sinh \left(m_{2} l\right)} A_{5}, \\
& A_{4}=\frac{\left(m_{1}^{2}-(s / v)^{2}\right)\left(\exp \left(-m_{2} l\right)-\exp (-(s / v) x l)\right)}{2\left(m_{1}^{2}-m_{2}^{2}\right) \sinh \left(m_{2} l\right)} A_{5} .
\end{aligned}
$$

Substituting $\bar{u}$ and $\bar{T}$ into equation (22), we obtain

$$
\begin{aligned}
\bar{\sigma}= & -\frac{s^{2}}{m_{1}} A_{1} e^{-m_{1} x}+\frac{s^{2}}{m_{1}} A_{2} e^{m_{1} x}+-\frac{s^{2}}{m_{2}} A_{3} e^{-m_{2} x}+\frac{s^{2}}{m_{2}} A_{4} e^{m_{2} x} \\
& -s v A_{5} \exp \left(-\frac{s}{v} x\right)-\frac{\bar{D}}{s} .
\end{aligned}
$$

\section{Laplace Transform Inversion}

We will apply a numerical technique to calculate the inversion of Laplace transform to get the physical fields of the problem in the actual field. To reverse the Laplace transform of various fields, we espouse a method based on the expansion of the Fourier series [31]. In this technique, any field $\bar{g}(x, s)$ in the inverse field of the Laplace transform is reversed in the physical domain using the relation

$$
g(x, t)=\frac{e^{c t}}{T_{1}}\left(\frac{\bar{g}(x, c)}{2}+\operatorname{Re} \sum_{k=1}^{N} \exp \left(\frac{i k \pi t}{T_{1}}\right) \bar{g}\left(x, c+\frac{i k \pi}{T_{1}}\right)\right)
$$

$$
2 t \geq T_{1} \geq 0,
$$

where $N$ is a sufficiently large integer which was chosen such that

$$
e^{c t} \operatorname{Re}\left(\exp \left(\frac{i k \pi t}{T_{1}}\right) \bar{g}\left(c+\frac{i k \pi}{T_{1}}\right)\right) \leq \varepsilon_{1},
$$

where $\varepsilon_{1}$ is a small positive and the parameter $c$ is positivefree as stated by Honig [31].

\section{Numerical Results and Discussion}

To illustrate the above results, the material constants of the piezoelectric rod were taken as follows given in Table 1 [8].

The numerical calculation and the discussion of the problem are carried out in two different groups for different positions of $x$ and time $t$. The first group is studying how the variations of displacement, temperature, and stress differ with various velocities of the heat source $v$ when the other parameters are constants. The second case shows the study of variations of different fields in models of thermoelasticity and the numerical results were also compared in different models. The last case is the study of the dependence of different physical distributions on the time when the parameter $v$ remains constant. The figures display that there are two separate phases in the history of electrical potential: before and after heat source leaves the rod. The effect of a heat source prevails in the first stage, while the relation between electrical power and displacement plays an important role in the second stage and contributes to the vibration of the electrical potential as shown in Figure 1.

Figures 2-4 display the variants of temperature, displacement, and pressure with various values of the parameter $v(v=1,2,3)$ in which we detect the following.

Figure 2 displays that when the parameter $v$ increases, there is an increase in the profile of temperature.

The distribution of temperature $T$ begins at its greatest value at $x=0$ and gradually decreases until the zero wave reaches the direction of propagation of the heat wave.

As Figure 2 shows, although still the wavefront of the simplified solution travels to the right end of the pole, the wave front is on its way back to the left end of the rod. This means that the thermal wave speed is greater than the simplified solution in the current solution. More 
TABLE 1: Physical constants of piezoelectric material.

\begin{tabular}{lc}
\hline Parameter & Value \\
\hline$K$ & $386 \mathrm{NK}^{-1} \mathrm{~s}^{-1}$ \\
$\mu$ & $3.86 \times 10^{10} \mathrm{Nm}^{-2}$ \\
$\rho$ & $8954 \mathrm{~kg} \mathrm{~m}^{-3}$ \\
$\alpha_{t}$ & $1.78 \times 10^{-5} \mathrm{~K}^{-1}$ \\
$T_{0}$ & $293 \mathrm{~K}$ \\
$\varepsilon$ & 0.0168 \\
$l$ & 5 \\
$\eta$ & 0.003887 \\
$Q_{0}$ & 10 \\
$D$ & $10^{-6}$ \\
$\alpha$ & 0.0104 \\
$\lambda$ & $7.76 \times 10^{10} \mathrm{Nm}^{-2}$ \\
\hline
\end{tabular}

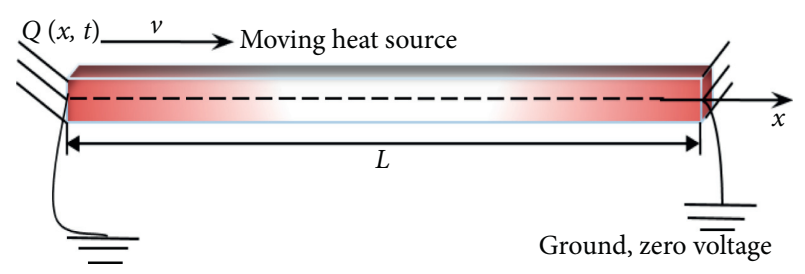

FIGURE 1: Schematic diagram for the piezoelectric finite rod.

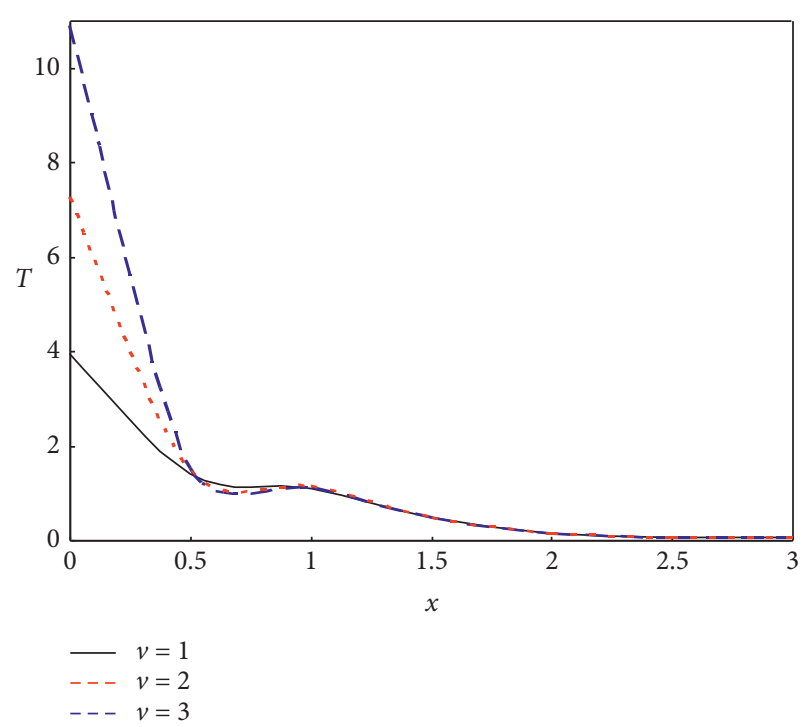

FIgURE 2: The variation of temperature distribution $T$ with moving heat source velocity $v$.

importantly, while in [32] the authors believed thermal wave fronts were present, they did not actually exist for a longer rod with $L=10$, as shown in [32].

From Figure 3, we can observe that the distribution of displacement $u$ decreases with increasing the parameter $v$. This results in a reduction in the density of thermal energy along the length of the rod.

It can also be found from Figure 3 that the displacement at $x=0,5$ is kept at a zero value, which corresponds to the boundary conditions of the problem where the piezoelectric rod is fixed.
The displacement variation $u$ increases monotonically and it reaches a peak value at another wave front.

From Figure 4, it is clear to us that the absolute stress decreases with increasing speed due to the decrease in the energy of the applied heat source along the axial of the rod.

The distributions of physical fields are graphically depicted in Figures 5-7 for different theories of thermal elasticity. This group illustrates the influence of two-phaselag parameters on the numerical results, and we observed a slight difference in the value of the distributions in the three models.

Figures $8-10$ are presented to illustrate the distributions of the physical fields at various values of time $t$ to show the influence of time in each field. We note the following:

The temperature profile increases with increasing value of time during certain periods.

The maximum values of the distributions of displacement and stress increase as time increases.

Finally, through Figures 2-10, it becomes clear that once the immediate time is given, the nonzero values of temperature, displacement, and thermal stress are only in a limited region and, beyond this area, gradually fade away. This explains why the heat dissipation speed is limited in the piezoelectric rod, as this is completely different as compared to the classical thermal models, which predict unlimited velocity. Due to the limited heat diffusion, the heat-disturbed region is limited when the appropriate time is given, resulting in the pressure and displacement caused by the heat also in a specific region.

From Figure 3, we can observe that the distribution of displacement $u$ decreases with increasing the parameter $v$. This results in a reduction in the density of thermal energy along the length of the rod.

It can also be found from Figure 3 that the displacement at $x=0,5$ is kept at a zero value which corresponds to the boundary conditions of the problem where the piezoelectric rod is fixed.

The displacement variation $u$ increases monotonically and it reaches a peak value at another wave front.

From Figure 4, it is clear to us that the absolute stress decreases with increasing speed due to the decrease in the energy of the applied heat source along the axial of the rod.

The temperature field stabilizes as the heat source moves away from the rod, contrary to the acceleration, electrical potential, and stress fields. There can, however, be some variations before the temperature achieves a constant value, depending on the thermal rest time.

The distributions of physical fields are graphically depicted in Figures 5-7 for different theories of thermal elasticity. This group illustrates the influence of two-phaselag parameters on the numerical results, and we observed a slight difference in the value of the distributions in the three models. The propagation of thermal waves is very sensitive to the speed of heat sources. Higher speed allows the thermal disturbance to spread more rapidly. The magnitudes of the nondimensional variables increase as the speed of the moving heat resource increases. As the largest values of curves indicate obviously, the effect of the thermal source transmission speed on all considered quantities is quite high. 


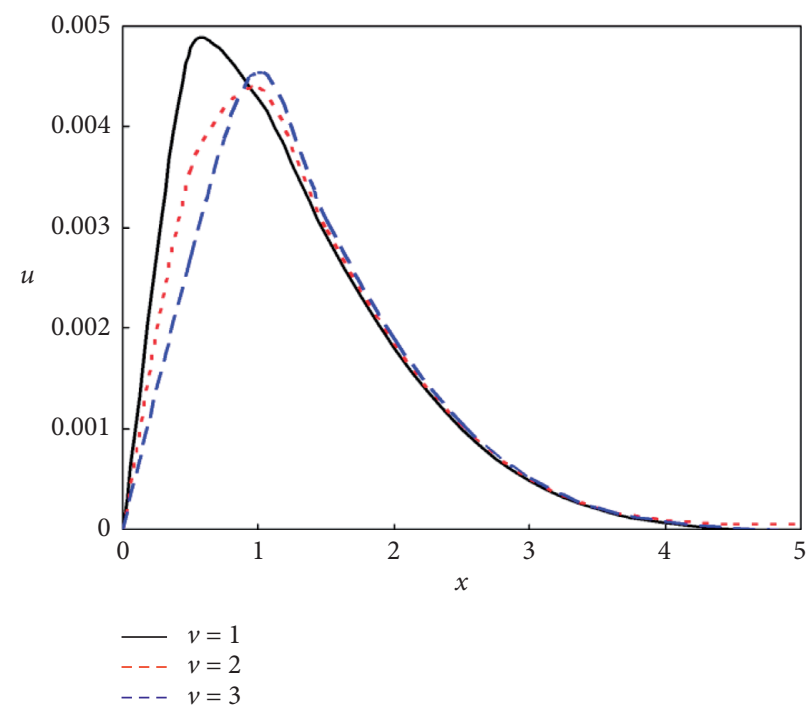

FIgURE 3: The variation of displacement distribution $u$ with moving heat source velocity $v$.

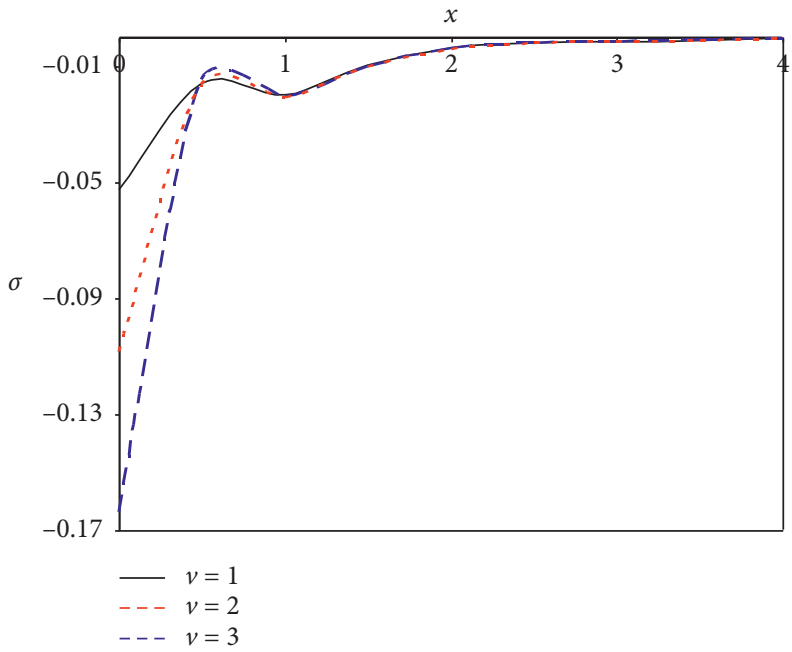

FIgURE 4: The variation of stress distribution $\sigma$ with moving heat source velocity $v$.

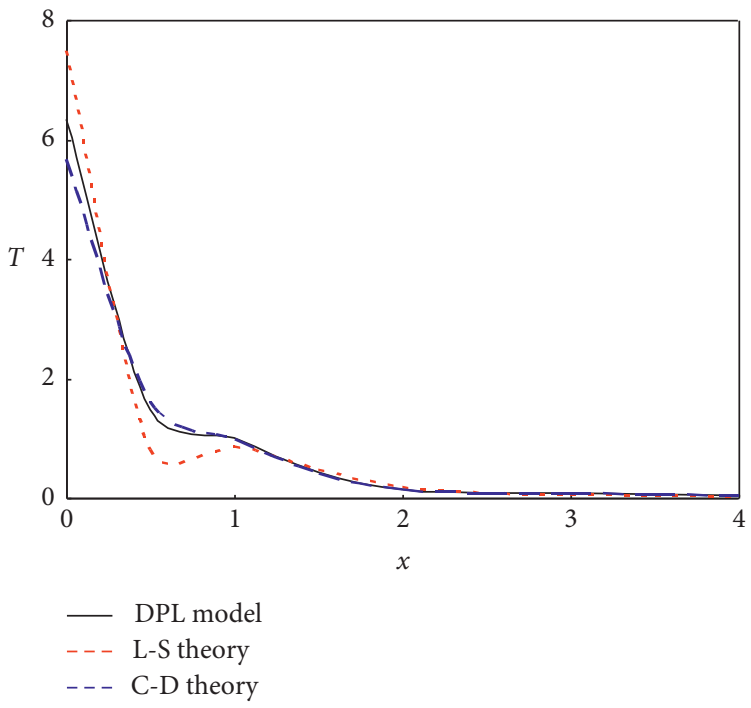

FIgURE 5: Temperature distribution for different theory of thermoelasticity. 


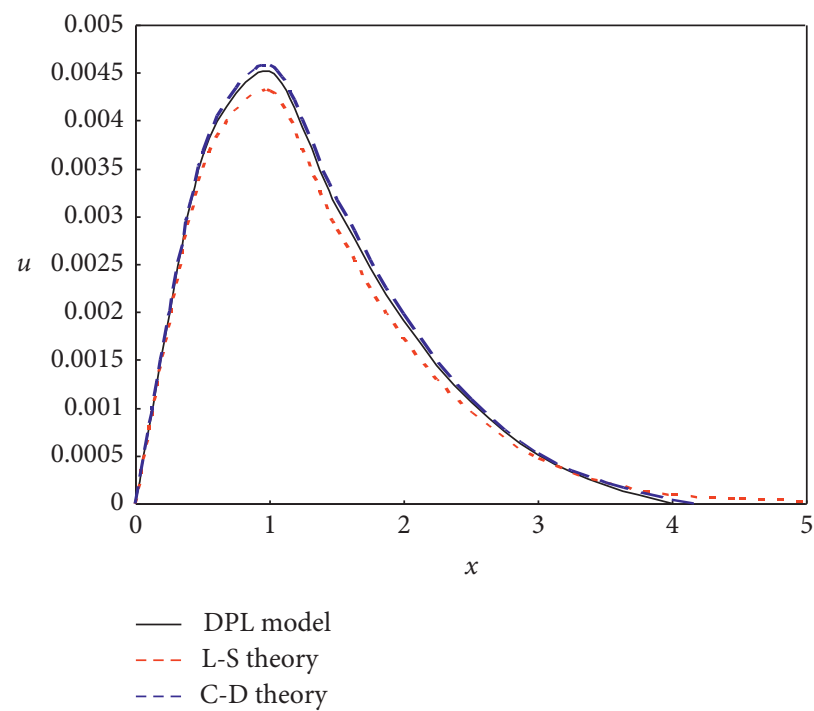

FIgURE 6: Displacement distribution for different theory of thermoelasticity.

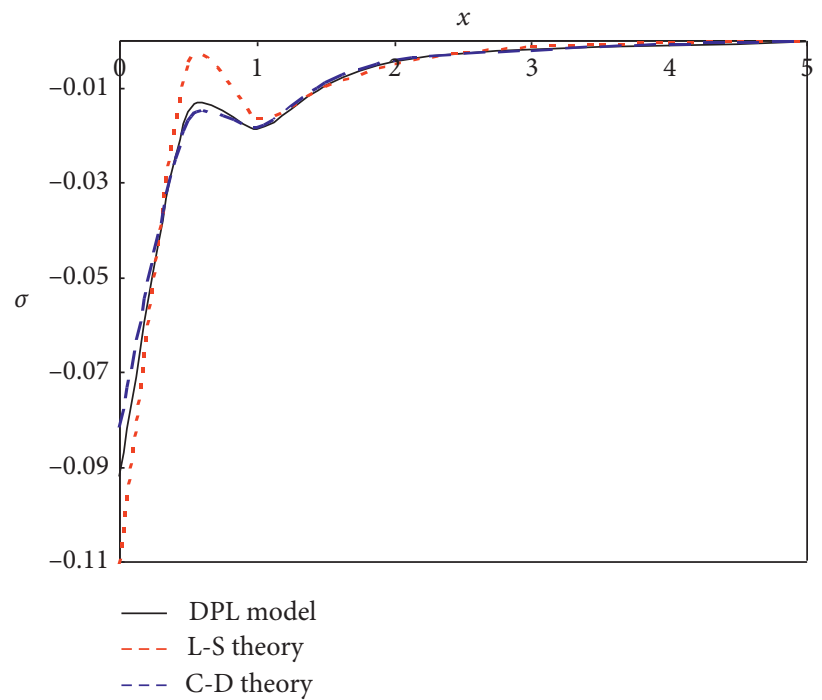

Figure 7: Stress distribution for different theory of thermoelasticity.

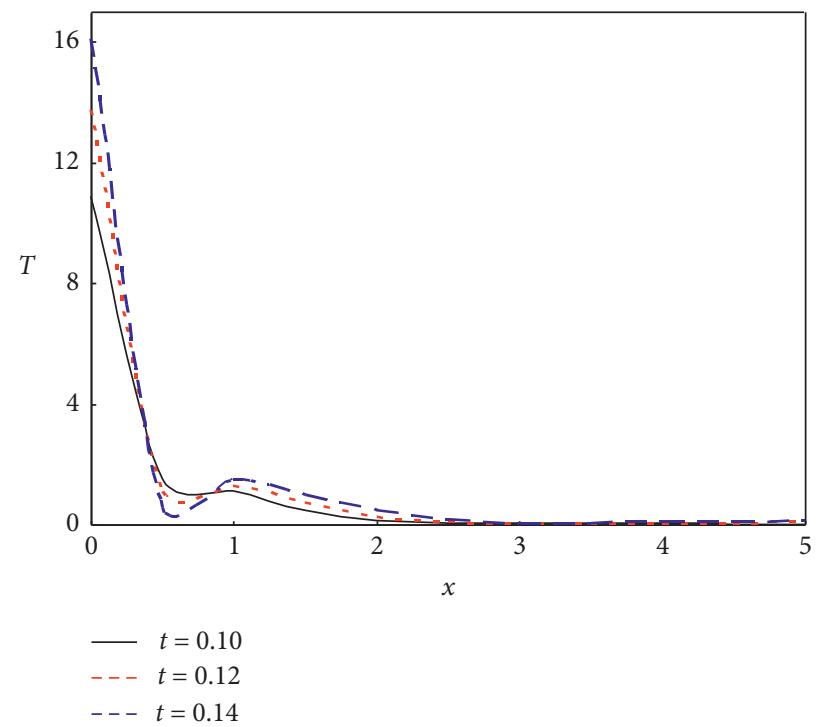

Figure 8: Temperature distribution for different values of time. 


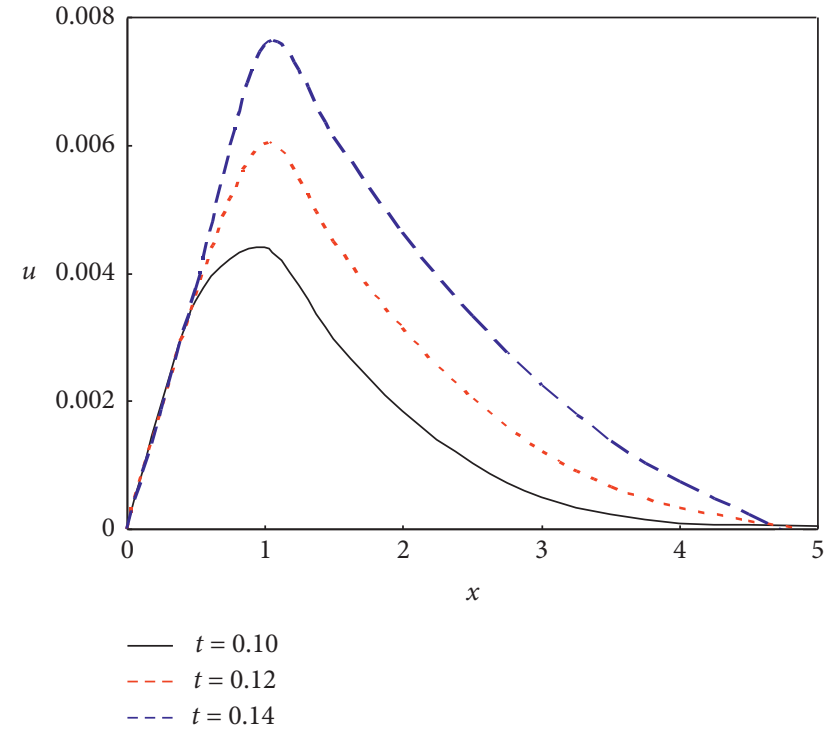

FIGURE 9: Displacement distribution for different values of time.

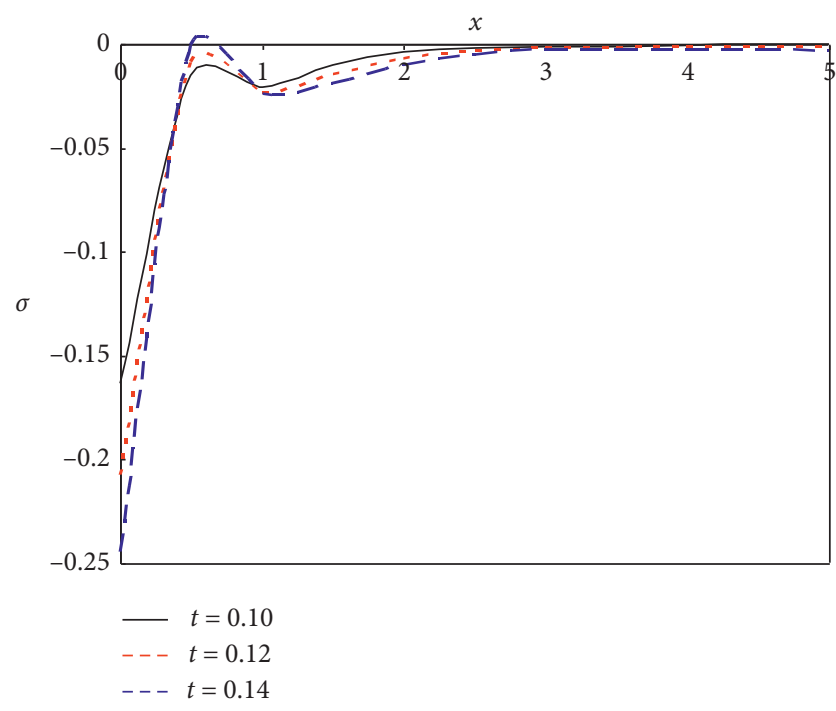

FIgURE 10: Thermal stress distribution for different values of time.

The influence of the heat source is an inherent consideration not to be overlooked in the evaluation of heat stress and fields at the heat source.

Figures $8-10$ are presented to illustrate the distributions of the physical fields at various values of time $t$ to show the influence of time in each field. We note the following:

The temperature profile increases with increasing value of time during certain periods.

The maximum values of the distributions of displacement and stress increase as time increases.

Finally, through Figures 2-10, it becomes clear that once the immediate time is given, the nonzero values of temperature, displacement, and thermal stress are only in a limited region and, beyond this area, gradually fade away. This explains why the heat dissipation speed is limited in the piezoelectric rod, as this is completely different as compared to the classical thermal models, which predict unlimited velocity. Due to the limited heat diffusion, the heat-disturbed region is limited when the appropriate time is given, resulting in the pressure and displacement caused by the heat also in a specific region.

\section{Conclusion}

The response of a thermoelastic piezoelectric rod exposed to a heat source is studied using the model of thermoelasticity with phase lags. The coupled governing equations of piezoelectricity and thermoelasticity are expressed. Based on the Laplace transform and an approximation inversion technique, the system of equations is solved. The results of the physical variables are displayed graphically and discussed.

From the graphs and theoretical discussion, we can conclude the following: With a comparison between the different models of thermoelasticity, that is, coupled theory (CD), Lord and Shulman (LS), and Tzou (DPL) models, there are differences in the results between different models.

The phenomenon of limited propagation speeds is manifested in all these forms. The heat wave interface is moving forward at a limited speed over time. This indicates that the mechanism of heat wave propagation in the case of generalized theories of thermoelasticity differs from that in the classical theory.

The values of temperature, exclusion, and nondimensional stress increase with the increase of the moving heat source. The value of $v$ has a significant impact on all areas of distribution in the case of the three theories.

\section{Data Availability}

The data are supported from the authors' published papers, and all the results are new.

\section{Conflicts of Interest}

The authors declare that they have no conflicts of interest.

\section{Acknowledgments}

This work was funded by the Deanship of Scientific Research (DSR), King Abdulaziz University, Jeddah, under Grant no. D-511-352-1441. The authors, therefore, gratefully acknowledge DSR for technical and financial support.

\section{References}

[1] H. W. Lord and Y. Shulman, "A generalized dynamical theory of thermoelasticity," Journal of the Mechanics and Physics of Solids, vol. 15, no. 5, pp. 299-309, 1967.

[2] A. E. Green and K. A. Lindsay, "Thermoelasticity," Journal of Elasticity, vol. 2, no. 1, pp. 1-7, 1972.

[3] A. E. Green and P. M. Naghdi, "Thermoelasticity without energy dissipation," Journal of Elasticity, vol. 31, no. 3, pp. 189-208, 1993. 
[4] D. Y. Tzou, "A unified field approach for heat conduction from macro- to micro-scales," Journal of Heat Transfer, vol. 117, no. 1, pp. 8-16, 1995.

[5] D. Y. Tzou, "Experimental support for the lagging behavior in heat propagation," Journal of Thermophysics and Heat Transfer, vol. 9, no. 4, pp. 686-693, 1995.

[6] D. Y. Tzou, Macro- to Microscale Heat Transfer: The Lagging Behavior, Taylor \& Francis, Washington, DC, USA, 1996.

[7] A. E. Abouelregal, "Fractional order generalized thermo-piezoelectric semi-infinite medium with temperature-dependent properties subjected to a ramp-type heating," Journal of Thermal Stresses, vol. 34, no. 11, pp. 1139-1155, 2011.

[8] A. E. Abouelregal, "On green and Naghdi thermoelasticity model without energy dissipation with higher order time differential and phase-lags," Journal of Applied Mathematics and Computational Mechanics, vol. 6, no. 3, pp. 445-456, 2020.

[9] A. E. Abouelregal and S. M. Abo-Dahab, "Dual phase lag model on magneto-thermoelasticity infinite nonhomogeneous solid having a spherical cavity," Journal of Thermal Stresses, vol. 35, no. 9, pp. 733-848, 2012.

[10] A. E. Abouelregal and S. M. Abo-Dahab, "Dual-phase-lag diffusion model for Thomson's phenomenon on electromagneto-thermoelastic an infinitely long solid cylinder," Journal of Computational and Theoretical Nanoscience, vol. 11, no. 4, pp. 1031-1039, 2014.

[11] S. Mondal, N. Sarkar, and N. Sarkar, "Waves in dual-phase-lag thermoelastic materials with voids based on Eringen's nonlocal elasticity," Journal of Thermal Stresses, vol. 42, no. 8, pp. 1035-1050, 2019.

[12] N. Sarkar and S. Mondal, "Thermoelastic interactions in a slim strip due to a moving heat source under dual-phase-lag heat transfer," ASME Journal Heat Transfer, vol. 141, no. 12, Article ID 124501, 2019.

[13] D. Li and T. He, "Investigation of generalized piezoelectricthermoelastic problem with nonlocal effect and temperaturedependent properties," Heliyon, vol. 4, no. 10, Article ID e00860, 2018.

[14] Y. Liu, J. Deng, Y. Ye, Z. Hou, Z. Duan, and S. Xu, "Piezoelectric thermoelastic dissipation research of piezoelectric harvester under different vibration," MATEC Web of Conferences, vol. 232, Article ID 04066, 2018.

[15] A. A. Kilany, S. M. Abo-Dahab, A. M. Abd-Alla, and A. N. Abd-alla, "Photothermal and void effect of a semiconductor rotational medium based on Lord-Shulman theory," Mechanics Based Design of Structures and Machines, pp. 1-14, 2020.

[16] Y. Abd-alla and T. H. He, "Dynamic response of a generalized piezoelectric-thermoelastic problem under fractional order theory of thermoelasticity," Mechanics of Advanced Materials and Structures, vol. 23, no. 10, pp. 1173-1180, 2016.

[17] M. I. A. Othman, Y. D. Elmaklizi, and E. A. A. Ahmed, "Effect of magnetic field on piezo-thermoelastic medium with three theories," Results in Physics, vol. 7, pp. 3361-3368, 2017.

[18] A. Taghizadeh and Y. Kiani, "Generalized thermoelasticity of a piezoelectric layer," Journal of Thermal Stresses, vol. 42, no. 7, pp. 863-873, 2019.

[19] N. S. Al-Huniti, M. A. Al-Nimr, and M. Naji, "Dynamic response of rod due to a moving heat source under the hyperbolic heat conduction model," Journal of Sound and Vibration, vol. 242, no. 4, pp. 629-640, 2011.

[20] R. D. Mindlin, "Equation of high frequency of thermo-piezoelectric, crystals plates Interactions in elastic solids," in
Proceedings of the International Conference on Sonar Sensors of Systems, 1979.

[21] W. Nowacki, "Some general theorems of thermopiezoelectricity," Journal of Thermal Stresses, vol. 1, no. 2, pp. 171-182, 1978.

[22] W. Nowacki, "Foundation of linear piezoelectricity," in Proceedings of the International conference on Sonar Sensors of Systems, 1979.

[23] D. S. Chandrasekharaiah, "A generalized linear thermoelasticity theory of piezoelectric media," Acta Mechanica, vol. 71, pp. 293-349, 1984.

[24] D. S. Chandrasekharaiah, "One-dimensional wave propagation in the linear theory of thermoelasticity without energy dissipation," Journal of Thermal Stresses, vol. 19, no. 8, pp. 695-710, 1996.

[25] S. K. Roychoudhuri and P. S. Dutta, "Thermo-elastic interaction without energy dissipation in an infinite solid with distributed periodically varying heat sources," International Journal of Solids and Structures, vol. 42, no. 14, pp. 4192-4203, 2005.

[26] S. M. Abo-Dahab, A. M. Abd-Alla, and A. A. Kilany, "Effects of rotation and gravity on an electro-magneto-thermoelastic medium with diffusion and voids by using the Lord-Shulman and dual-phase-lag models," Applied Mathematics and Mechanics, vol. 40, no. 8, pp. 1135-1154, 2019.

[27] S. S. Nanthakumar, T. Lahmer, X. Zhuang, G. Zi, and T. Rabczuk, "Detection of material interfaces using a regularized level set method in piezoelectric structures," Inverse Problems in Science and Engineering, vol. 24, no. 1, pp. 153176, 2015.

[28] E. Samaniego, C. Anitescu, S. Goswami et al., "An energy approach to the solution of partial differential equations in computational mechanics via machine learning: concepts, implementation and applications," Computer Methods in Applied Mechanics and Engineering, vol. 362, Article ID 112790, 2020.

[29] K. M. Hamdia, H. Ghasemi, X. Zhuang, N. Alajlan, and T. Rabczuk, "Sensitivity and uncertainty analysis for flexoelectric nanostructures," Computer Methods in Applied Mechanics and Engineering, vol. 337, pp. 95-109, 2018.

[30] E. M. Khalil, S. M. Abo-Dahab, and A. A. Kilany, "Electromagnetic field and initial stress on a photothermal semico nducting voids medium under thermoelasticity theories," Mathematical Methods in the Applied Sciences, pp. 1-21, 2020.

[31] G. Honig and U. Hirdes, "A method for the numerical inversion of Laplace transforms," Journal of Computational and Applied Mathematics, vol. 10, no. 1, pp. 113-132, 1984.

[32] T. He, L. Cao, and S. Li, "Dynamic response of a piezoelectric rod with thermal relaxation," Journal of Sound and Vibration, vol. 306, no. 3-5, pp. 897-907, 2007.

[33] S. M. Abo-Dahab, S. Z. Rida, R. A. Mohamed, and A. A. Kilany, "Rotation, initial stress, gravity and electromagnetic field effect on $\mathrm{P}$ wave reflection from stress-free surface elastic half-space with voids under three thermoelastic models," Journal of Mechanics and Mechanical Engineering, vol. 22, no. 1, pp. 313-328, 2018.

[34] S. M. Abo-Dahab, A. A. Kilany, E. A.-B. Abdel-Salam, and A. Hatem, "Fractional derivative order analysis and temperature-dependent properties on $\mathrm{p}$ - and SV-waves reflection under initial stress and three-phase-lag model," Results in Physics, vol. 18, Article ID 103270, 2020.

[35] S. M. Abo-Dahab, A. A. Kilany, M. N. M. Allam, R. A. Mohamed, and S. Z. Rida, "Influence of several fields on Rayleigh waves propagation in a fiber-reinforced orthotropic 
half-space material under four thermoelastic models," Waves in Random and Complex Media, pp. 1-24, 2020.

[36] S. M. Abo-Dahab, A. M. Abd-Alla, and A. A. Kilany, "Electromagnetic field in fiber-reinforced micropolar thermoelastic medium using four models," Journal of Ocean Engineering and Science, vol. 5, no. 3, pp. 230-248, 2020.

[37] S. M. Abo-Dahab, A. M. Abd-Alla, A. A. Kilany, and M. Elsagheer, "Effect of rotation and gravity on the reflection of P-waves from thermo-magneto-microstretch medium in the context of three phase lag model with initial stress," Microsystem Technologies, vol. 24, no. 8, pp. 3357-3369, 2018.

[38] H. Alotaibi, S. M. Abo-Dahab, H. R. Abdlrahim, and A. A. Kilany, "Fractional calculus of thermoelastic $p$-waves reflection under influence of gravity and electromagnetic fields," Fractals, vol. 28, no. 8, 17 pages, Article ID 2040037, 2020.

[39] A. M. Abd-Alla, S. M. Abo-Dahab, and A. A. Kilany, "Effect of several fields on a generalized thermoelastic medium with voids in the context of Lord-Shulman or dual-phase-lag models," Mechanics Based Design of Structures and Machines, pp. 1-24, 2020. 